\title{
LUT
}

University

\section{Numerical Treatment of Singularity in Hydraulic Circuits Using Singular Perturbation Theory}

Kiani Oshtorjani Mehran, Mikkola Aki, Jalali Payman

This is a Author's accepted manuscript (AAM) version of a publication published by IEEE

in IEEE/ASME Transactions on Mechatronics

DOI: $\quad$ 10.1109/TMECH.2018.2876157

Copyright of the original publication: (C) 2018 IEEE

Please cite the publication as follows:

Kiani Oshtorjani, M., Mikkola, A., Jalali, P. (2018). Numerical Treatment of Singularity in Hydraulic Circuits Using Singular Perturbation Theory. IEEE/ASME Transactions on Mechatronics, vol. 24, iss. 1, pp. 145-153. DOI: 10.1109/TMECH.2018.2876157

This is a parallel published version of an original publication. This version can differ from the original published article. 


\title{
Numerical treatment of singularity in hydraulic circuits using singular perturbation theory
}

\author{
Mehran Kiani ${ }^{1,3}$, Aki Mikkola ${ }^{2,3}$, and Payman Jalali ${ }^{1,3}$ \\ ${ }^{1}$ Laboratory of Thermodynamics, School of Energy Systems, Lappeenranta University of Technology, Lappeenranta, Finland \\ ${ }^{2}$ Department of Mechanical Engineering, School of Energy Systems, Lappeenranta University of Technology, Lappeenranta, Finland \\ ${ }^{3}$ SIM-Platform, Lappeenranta University of Technology, Lappeenranta, Finland
}

\begin{abstract}
Hydraulic cylinders and motors are commonly used in actuator systems of mobile machines and industrial applications. Hydraulic actuators have the ability to offer large amounts of energy density. To analyze the dynamic behavior of a hydraulic actuator system, the hydraulic circuit is generally divided into volumes in which pressure is assumed to be equally distributed. Pressure within these volumes can be predicted by employing first order differential equations. To achieve an accurate numerical presentation of a hydraulic circuit, pressures within very small volumes must often be calculated. The small volumes involved in the hydraulic circuits make the models numerically stiff and consequently a very small integration time step is required. In this paper, singular perturbation theory, which can be applied to problems in which a small value appears with singularity effects, is implemented as a computationally-efficient method for modeling hydraulic circuits. The implementation is performed in two stages: derivation of the perturbed equations; and modification of their effects. It is shown that usage of a perturbation theory based approach alleviates problems associated with small volumes in hydraulic system modeling and substantially enhances computational efficiency, thus facilitating real-time simulation.
\end{abstract}

Index Terms-Hydraulic system, Runge-Kutta integration, Perturbation theory, Boundary-layers stability analysis, Time and frequency responses.

\section{INTRODUCTION}

$\mathbf{P}$ RODUCTS and machinery in the mechanical and electromechanical industry, such as mobile machines, are complex devices and their development requires knowledge of different areas of technology, including applied mechanics, and the operation of actuation and control systems. The various subsystems involved in a mechanical device interact closely. The actuators are mounted on the mechanism and produce forces acting on the mechanism, which are converted into the constrained motion of the original system of bodies.

In product development, hydraulic actuators and associated hydraulic circuits are often designed with the help of computer simulation. To this end, lumped fluid theory can be used to form differential equations for volumes where pressure is assumed to be equally distributed. In practice, the usage of lumped fluid theory leads to a situation in which small volumes exist in the locations of the pressure calculations. This model has been used frequently in literature [1], [2], [3] as well as the commercial packages such as AMESim to model various hydraulic components as mentioned in [4]. However, several challenging issues in computational time and stability remains for simulation of hydraulic circuit itself and its controller. For instance, Ref. [1] has indicated that a large value of bulk modulus can easily overwhelm the effects of controller parameters if there is a poor controller design.

Bowns and Wangs [5] reported that there is a difficulty in simulation of hydraulic pipe systems with small volumes. They noticed that if volumes of one or several pipes are small, the required simulation time steps become very small.

Manuscript received xxx; revised xxx. Corresponding author: Mehran Kiani (email: mehran.kiani@lut.fi).
To overcome this problem, they proposed iterative models, however, these iterative methods are computationally costly and similar to applying small time steps in the integration.

Piche and Ellman [6] also noticed that different volumes are difficult to solve forward in time, because the corresponding ordinary differential equations of the model are numerically stiff. They investigated the accuracy and numerical stability of different two-stage semi-implicit Runge-Kutta methods. They used the time step of $2 \mathrm{~ms}$ in all simulations and proposed that L-stable is the best-suited approach for the application under investigation. Borutzky et al. [7] proposed an empiricallyobtained polynomial function for the orifice volumetric flow rate which has a smooth transition between laminar and turbulent flow regimes. With this approach, singularities can be avoided when the pressure difference approaches zero.

From the modeling point of view, a mechanical system actuated by hydraulics behaves differently at small and large time scales. In hydraulic circuit modeling where the large bulk modulus is divided into small volumes, small time scales will appear. This small time scale increases the computational costs of simulations. As reported by Pfeiffer and Borchsenius [8], the high computational costs are mainly due to the mathematical representation of the hydraulic systems used in the simulations, which are non-linear, first order differential equations.

Esque et al. [9] studied the real-time simulation of a hydraulic crane using an L-stable Rosenbrock integration scheme. They remarked that the maximum time step of integration is imposed by stability and computational time criteria. Based on these two factors, they selected the time step as $0.1 \mathrm{~ms}$ in their application.

To overcome the stiffness of the hydraulic equations, the 
time step can be reduced. To this end, some values as small as $0.001 \mathrm{~ms}$ have been reported [10]. The problem gets more difficult if the hydraulic circuit is coupled with a mechanical system. There are two common approaches for dealing with a system of a combined mechanical model and hydraulic circuits. The first is called the unified approach [11], [12], in which the hydraulic equations and equations for the mechanical system are combined as a single system in which the integration scheme and the time step are identical in both subsystems. In the multi-rate integration approach [13], [14], different time steps are used for the mechanical system and hydraulics. The multi-rate integration method can be computationally less costly as the hydraulic simulation usually needs the smaller time step [15].

Ylinen et al. [16] have developed a monolithic algorithm in which the hydraulic system is coupled with the mechanical system. The whole system is solved with a single step Crank Nicolson scheme (trapezoidal rule). They used a time step of $1 \mathrm{~ms}$ for the coupled system. However, the predictor-corrector used in their work would double the computational cost.

Bauchau, and Liu [17] used a multi-integration algorithm to solve a finite element based mechanical system coupled with hydraulic equations. They used a time step of $0.1 \mathrm{~ms}$ for the structural dynamics analysis, and a four step Runge-Kutta integrator with a time step 48 times smaller than the structural solver in the hydraulic integration.

Naya et al. [18] compared the unified and co-integration approaches. They noted that the unified scheme is computationally 20 percent more costly than the simplified simulation that considers only the mechanical system. However, they suggested use of the simplified simulation only when the computational efficiency has priority, because the response of this approach shows many oscillations around the correct answers. It is important to note that these oscillations make it difficult to use this method for many applications, especially for real-time applications. In their study, the utilized time step was $10 \mathrm{~ms}$ for the unified scheme, $0.2 \mathrm{~ms}$ for a co-integration scheme with a forward Euler integrator, and $5 \mathrm{~ms}$ for a cointegration scheme with a trapezoidal integrator.

In summary, the behavior of hydraulic circuits resembles some other engineering systems that suffer from numerical stiffness [19]. Accordingly, from the mathematical point of view, singular perturbation theory and, practically, Tikhonovs theorem may be considered permissible as an approach to address numerical problems in such systems.

The objective of this paper is to investigate application of the singular perturbation theory to remove the singularities and numerical difficulties associated with simulation of hydraulic systems. The response of a typical hydraulic system is investigated before and after applying the perturbation theory based calculations to find out how, and to what extent, use of the theory is able to improve the numerical efficiency of formulations associated to the hydraulic model. The paper is organized as follows. Section II describes the hydraulic formulation, which is followed by a brief introduction to singular perturbation theory in section III. The numerical model of the hydraulic system is explained in section IV, where the mathematical equations are first described and the perturbation method then applied to handle singularity. Results are presented and discussed in section V. The final section gives a brief summary and presents conclusions.

\section{HYDRAULIC FORMULATION}

The theory of lumped fluids is often used in modeling of hydraulic systems. In the lumped fluid theory, the hydraulic circuit is divided into volumes in which the pressure is assumed to be evenly distributed. Differential equations are formed for the volumes with which the pressure of the system at a certain time can be solved directly or indirectly. Different volumes are assumed to be separated by throttling through which the fluid can flow. The directional, pressure and flow control valves as well as long pipelines used in real systems are replaced by throttles that control the flow rate between the different volumes.

The pressure in a volume of the hydraulic circuit can be calculated using differential equation as follows [20]:

$$
\frac{d p}{d t}=\frac{B_{e}}{V}\left(Q_{\text {in }}-Q_{\text {out }}-\frac{d V}{d t}\right)
$$

where $p$ is the pressure, $B_{e}$ is the effective bulk modulus, $V$ is the volume, $Q_{\text {in }}$ and $Q_{\text {out }}$ are the incoming and outgoing volumetric flow rates, and $d V / d t$ is the time rate changes of volume $V$. The effective bulk modulus represents the bulk modulus of the fluid by taking into account the effects of the flexibility of the container and dissolved air [21].

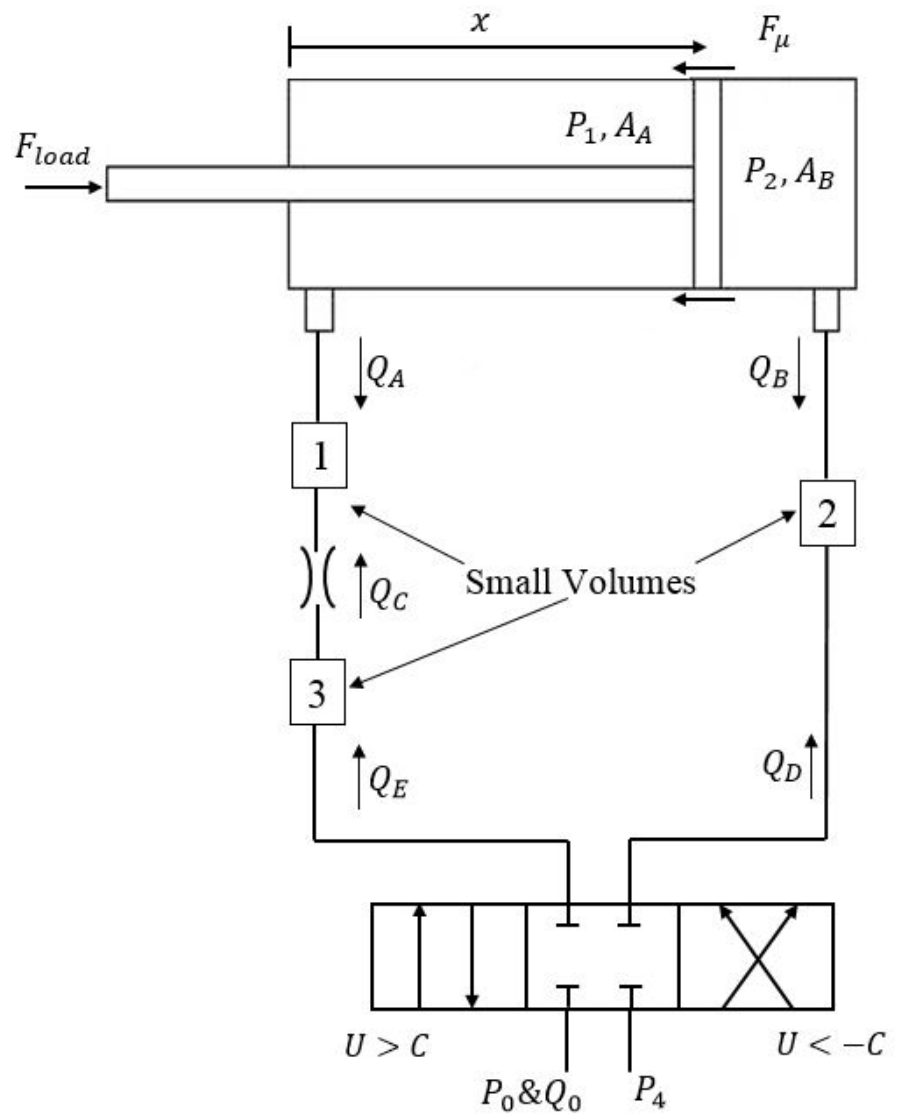

Fig. 1: The hydraulic circuit studied. 
TABLE I: The set values for different quantities.

\begin{tabular}{ccccccc}
\hline$A_{A} / m^{2}$ & $A_{B} / m^{2}$ & $B_{i} /$ Pa(Bulk modulus $)$ & $V_{1} / m^{3}$ & $V_{2} / m^{3}$ & $V_{3} / m^{3}$ & $P_{0} /$ Pa(Pump pres. $)$ \\
\hline 0.0122718 & 0.084234 & $70000.0 \sqrt{P_{i}}$ & 0.0061359 & 0.042117 & 0.005 & $10^{7}$ \\
\hline \multicolumn{7}{c}{} \\
\hline$C_{C}$ & $C_{P V}$ & $F_{\text {load }} / N($ External force $)$ & $C_{T V}$ & $m / k g$ & $C$ & $P_{4} /$ Pa(Tank pres. $)$ \\
\hline $5 \times 10^{-5}$ & $10^{-7}$ & -5000 & $8 \times 10^{-8}$ & 50.0 & $10^{-4}$ & $2 \times 10^{5}$ \\
\hline
\end{tabular}

TABLE II: The flow rate relations used in the calculations.

\begin{tabular}{cccc}
\hline & $U<-C$ & $-C<U<C$ & $U>C$ \\
\hline \hline$Q_{A}$ & $-\dot{x} A_{A}$ & $-\dot{x} A_{A}$ & $-\dot{x} A_{A}$ \\
$Q_{B}$ & $\dot{x} A_{B}$ & $\dot{x} A_{B}$ & $\dot{x} A_{B}$ \\
$Q_{C}$ & $C_{C} \sqrt{P_{1}-P_{3}} \operatorname{Step}\left(P_{3}-P_{1}\right)$ & $C_{C} \sqrt{\left|P_{1}-P_{3}\right|}$ Step $\left(P_{3}-P_{1}\right)$ & $C_{C} \sqrt{P_{3}-P_{1}}$ Step $\left(P_{3}-P_{1}\right)$ \\
$Q_{D}$ & $U C_{P V} \sqrt{P_{0}-P_{2}} S t e p\left(P_{2}-P_{0}\right)$ & 0 & $U C_{T V} \sqrt{P_{2}-P_{4}}$ Step $\left(P_{4}-P_{2}\right)$ \\
$Q_{E}$ & $U C_{T V} \sqrt{P_{3}-P_{4}} S t e p\left(P_{3}-P_{4}\right)$ & 0 & $U C_{P V} \sqrt{P_{0}-P_{3}}$ Step $\left(P_{0}-P_{3}\right)$ \\
\hline
\end{tabular}

The volumetric turbulent flow rate $Q_{t}$ in a throttle can be written as [7]:

$$
Q_{t}=C_{d} A_{t} \sqrt{\frac{2\left(P_{3}-P_{1}\right)}{\rho}}
$$

where $C_{d}$ is the discharge coefficient, $P_{1}$ and $P_{3}$ are pressures at both sides of throttle valve, $A_{t}$ is the cross-sectional area of the valve, and $\rho$ is the density of the fluid, and. In this study, the volumetric flow rate is described using semi-empirical methods in which the parameters of the valve can be obtained from measurements [22].

A hydraulic cylinder can be modeled based on its dimensions and the input pressures. The model of the cylinder can be formed based on a diagram as shown in Fig. 1. The hydraulic cylinder volume is related to the cylinder stroke as:

$$
\begin{array}{r}
V_{\text {in }}=x A_{A} \\
V_{\text {out }}=(l-x) A_{B}
\end{array}
$$

where $A_{A}$ and $A_{B}$ are the surface area of the front side of the piston, and the area of the rod side of the piston, respectively, and 1 is the maximum stroke length. The volumetric flow rates produced based on the piston motion can be written as:

$$
\begin{gathered}
Q_{\text {in }}=\dot{x} A_{A} \\
Q_{\text {out }}=-\dot{x} A_{B}
\end{gathered}
$$

The force $F_{s}$ produced by the cylinder can be written as:

$$
F_{s}=P_{1} A_{A}-P_{2} A_{B}+F_{\text {load }}-F_{\mu}
$$

where $F_{\text {load }}$ is the load force, $F_{\mu}$ is the friction force, and $P_{1}$ and $P_{2}$ are the pressure values in the cylinder chambers.

\section{Singular PERTURBATION THEORY}

In this section, the concept of singular perturbation theory is briefly introduced. Considering the state variables of $[\phi, \psi]$, the singular system can be represented by the following relations:

$$
\begin{aligned}
\dot{\phi}=f(\phi, \psi, t, \varepsilon) & \phi\left(t_{0}\right)=\zeta(\varepsilon) \\
\dot{\psi}=g(\phi, \psi, t, \varepsilon) & \psi\left(t_{0}\right)=\xi(\varepsilon)
\end{aligned}
$$

where $\phi \in R^{m}$ and $\psi \in R^{n}$, and $\varepsilon$ is an infinitesimal parameter.
The quasi-steady state model of the above system is [23]:

$$
\begin{array}{r}
\dot{\phi}=f(\phi, h(t, \phi), t, \varepsilon) \\
\bar{\psi}(t)=h(t, \bar{\phi})
\end{array}
$$

Note that the dot represents the time derivative, and the overbar indicates the perturbed variables. Based on Tikhonovs theorem [24] in singular perturbation theory, Wang et al. [25] stated the following strategy. Assume the functions $f$, $g$ to be smooth enough, and the boundary-layers model is exponentially stable. Also, assume the reduced-order system (Eq. 8a) is exponentially stable, and has unique solutions on a convex set. Then, a positive constant $\varepsilon^{*}$ can be found in a way that for every $0<\varepsilon<\varepsilon^{*}$, the system (Eq. 7) has a unique solution for $[t, \infty)$, and $\phi(t, \varepsilon)-\bar{\phi}(t)=O(\varepsilon)$. Moreover, for any given $t_{b}>t_{0}$, there is $0<\varepsilon<\varepsilon^{*}$ which satisfies $\psi(t, \varepsilon)-h(t, \bar{\phi})=O(\varepsilon)$, and holds uniformly for $t \in\left[t_{b}, \infty\right)$ [25], [26], [27].

In other words, it is possible to reduce the system of equations (7) into equations (8) while maintaining the accuracy as the corresponding errors on the response of system (8) are in the order of $O(\varepsilon)$. This theory is applied to remove the singularities associated with the hydraulic systems.

\section{NUMERICAL MODEL OF THE HYDRAULIC SYSTEM}

In this section, the hydraulic circuit illustrated in Fig. 1 is introduced. First, in section IV-A, the mathematical model of the system is presented, then perturbation theory is applied to the system in section IV-B.

\section{A. Mathematical model of the system}

In this section, the hydraulic system with two valves illustrated in Fig. 1 is modeled. The governing equations are first presented, followed by development of the model in the state space when the control signal is greater than $C$, i.e., $U>C$.

\section{1) Physical model}

Here, the mathematical formulation of the hydraulic circuit is presented. All constant parameters used are summarized in Table I. The governing equations can be written as:

$$
\dot{P}_{1}=\frac{B_{1}}{V_{1}}\left(Q_{A}+Q_{C}\right)
$$




$$
\begin{gathered}
\dot{P}_{2}=\frac{B_{2}}{V_{2}}\left(Q_{B}+Q_{D}\right) \\
\dot{P}_{3}=\frac{B_{2}}{V_{2}}\left(Q_{E}-Q_{C}\right) \\
\ddot{x}=\frac{1}{m}\left(P_{1} A_{A}-P_{2} A_{b}\right)+\frac{F_{\text {load }}}{m}
\end{gathered}
$$

where, $B_{i}$ represents the bulk modulus, and $V_{i}$ is the volumes for $i=1,2,3$. The relations of volumetric flow rates are obtained as presented in Table II in which pressures are embedded. Note that there are several parameters apperaing in this table which their values are presented in Table I. The Step function used in Table II is defined in Appendix A.

Assuming an ideal directional valve (where the flow, friction and pressure forces have no effect on the valve spool position), $U$ represents the position of the spool whose model is given in Appendix A. Hence, there are three positions for a $4 / 3$ directional valve (4 ways, and 3 positions), which are identified with parameter $C$.

\section{2) State-space representation}

If the hydraulic system is represented in the state space, the calculations associated with the boundary layers stability analysis, presented in section IV-B1, will be simpler and more efficient. The state variables for the problem under investigation are defined in Table III.

TABLE III: State Variables.

\begin{tabular}{cc}
\hline$X_{1}=\sqrt{P_{3}-P_{1}}$ & $X_{3}=\sqrt{P_{0}-P_{3}}$ \\
\hline$X_{2}=\sqrt{P_{2}-P_{4}}$ & $X_{4}=\sqrt{\dot{x}}$ \\
\hline
\end{tabular}

The first state equation, i.e. Eq. (13), can be derived by getting derivative of first state variable $X_{1}$ and considering Eqs. (9)-(12). The final form of state equations can be obtained as:

$$
\begin{gathered}
2 X_{1} \dot{X}_{1}=\frac{B_{1}}{V_{1}} A_{A} X_{4}^{2}+\frac{B_{3}}{V_{3}} U C_{P V} X_{3} \\
-\left(\frac{B_{1}}{V_{1}}+\frac{B_{3}}{V_{3}}\right) C_{C} X_{1} \\
2 X_{2} \dot{X}_{2}=\frac{B_{2}}{V_{2}} A_{B} X_{4}^{2}+\frac{B_{2}}{V_{2}} U C_{T V} X_{2}-\dot{P}_{4} \\
2 X_{3} \dot{X}_{3}=-\frac{B_{3}}{V_{3}} A_{B} X_{4}^{2}+\frac{B_{3}}{V_{3}} U C_{C} X_{1}+\dot{P}_{0} \\
2 X_{4} \dot{X}_{4}=-\frac{A_{A}}{m} X_{1}^{2}-\frac{A_{A}}{m} X_{3}^{2}-\frac{A_{B}}{m} X_{2}^{2}+\frac{F_{\text {load }}}{m} \\
+\frac{A_{A}}{m} P_{0}-\frac{A_{B}}{m} P_{4}
\end{gathered}
$$

These equations introduce a multi-input multi-output nonlinear system with the nonlinearities in the form of polynomial terms. If the control signal is negative, the state variables are defined as given in Table III. However, the governing equations will be different from Eqs. (13)-(16).

\section{B. Perturbation theory applied to mathematical equations}

In this section, the perturbation theory is applied to the hydraulic system. As mentioned in section II, perturbation theory is applied to the desired equation. Equations (9) to (11) can be rewritten as:

$$
\begin{aligned}
& \varepsilon \dot{P}_{1}=\left(Q_{A}+Q_{C}\right) \\
& \varepsilon \dot{P}_{2}=\left(Q_{B}+Q_{D}\right) \\
& \varepsilon \dot{P}_{3}=\left(Q_{E}-Q_{C}\right)
\end{aligned}
$$

where $\varepsilon$ is determined by the small volume divided by the effective bulk modulus, which is lowered by the entrained air [17], [28]. It is often observed when a hydraulic system is turned on after a period of shutdown, which allows air to collect in the system. However, it can be assumed that the effective bulk modulus is a constant or changes slowly under normal working conditions since the entrained air in the system has a relatively stable level [25].

\section{1) Boundary layers stability analysis}

The small values for some parameters appearing in the governing differential equations are important. If the small parameters are set to zero, and the differential equations are then solved, the results could differ from the exact solution of the differential equations containing the small parameter. In some systems, these two solutions are the same; such systems are usually called as regular systems. However, if the two solutions differ, the system is referred as a singular system.

In singular systems, two distinct solutions are obtained if the mathematical limits are calculated by approaching the small parameter to zero before and after solving the equations.

In these systems, the infinitesimal parameter is multiplied by the highest derivative term which usually makes a rapid variation in the small time scales [28]. This region is known as the boundary layer, whose stability puts forward a criterion for using singular perturbation theory.

To this end, the following variables are defined:

$$
\begin{gathered}
y_{1} \triangleq P_{1}-\bar{P}_{1}=-\frac{1}{C_{C}^{2}}\left(C_{C}^{2} X_{1}^{2}-A_{A}^{2} X_{4}^{4}\right) \\
y_{2} \triangleq P_{2}-\bar{P}_{2}=-\frac{1}{U C_{T V}^{2}}\left(U^{2} C_{T V}^{2} X_{2}^{2}-A_{B}^{2} X_{4}^{4}\right) \\
y_{3} \triangleq P_{3}-\bar{P}_{3}=\frac{X_{1}^{2}-\alpha X_{3}^{2}}{1+\alpha}
\end{gathered}
$$

where, the overbar represents the perturbed variable. Taking the derivative of Eq. (20) gives:

$$
\varepsilon \dot{y}_{1}=\varepsilon \frac{d y_{1}}{d t}=\frac{d y_{1}}{d \tau}=\varepsilon \dot{P}_{1}-\varepsilon \bar{P}_{1}
$$

Using Eq. (17), the following relation can be achieved when $\varepsilon \longrightarrow 0$ :

$$
\frac{d y_{1}}{d \tau}=\varepsilon \dot{P}_{1}=C_{C} X_{1}-A_{A} X_{4}^{2}
$$

On the other hand,

$$
\begin{aligned}
-C_{C}^{2} y_{1}=\left(C_{C}^{2} X_{1}^{2}-A_{A}^{2} X_{4}^{4}\right) & =\left(C_{C} X_{1}-A_{A} X_{4}^{2}\right) \\
& \times\left(C_{C} X_{1}+A_{A} X_{4}^{2}\right)
\end{aligned}
$$


If $\left(C_{C} X_{1}-A_{A} X_{4}^{2}\right)>0$, and $\left(C_{C} X_{1}+A_{A} X_{4}^{2}\right)>1$ then $-C_{C}^{2} y_{1}>\left(C_{C} X_{1}-A_{A} X_{4}^{2}\right)$, so:

$$
0<\frac{d y_{1}}{d \tau}<-C_{C}^{2} y_{1}
$$

Assuming $y_{1}=B e^{A t}$ :

$$
0<B A<-C_{C}^{2} B
$$

As a result, $\mathrm{B}$ must be negative, so $\mathrm{A}$ will be negative too.

Now, if $\left(C_{C} X_{1}-A_{A} X_{4}^{2}\right)<0$, and $\left(C_{C} X_{1}+A_{A} X_{4}^{2}\right)>1$ then $-C_{C}^{2} y_{1}<\left(C_{C} X_{1}-A_{A} X_{4}^{2}\right)$, and,

$$
-C_{C}^{2} y_{1}<\frac{d y_{1}}{d \tau}<0
$$

Again, assuming $y_{1}=B e^{A t}$ leads to:

$$
-C_{C}^{2} B<B A<0
$$

Here, $B$ must be positive; hence, $A$ will be negative. In this problem, following $\left(C_{C} X_{1}+A_{A} X_{4}^{2}\right)>1$ holds. However, it can be demonstrated mathematically that for the condition of $0<\left(C_{C} X_{1}+A_{A} X_{4}^{2}\right)<1$, the boundary layers model of Eq. (20) is exponentially stable.

The time derivative of Eq. (21) yields:

$$
\varepsilon \dot{y}_{2}=\varepsilon \frac{d y_{2}}{d t}=\frac{d y_{2}}{d \tau}=\varepsilon \dot{P}_{2}-\varepsilon \bar{P}_{2}
$$

Using Eq. (18) when $\varepsilon \longrightarrow 0$ the following relation can be obtained:

$$
\frac{d y_{2}}{d \tau}=\varepsilon \dot{P}_{2}=U C_{T V} X_{2}+A_{B} X_{4}^{2}
$$

On the other hand:

$$
\begin{array}{r}
U C_{T V}^{2} y_{2}=\left(U^{2} C_{T V}^{2} X_{2}^{2}-A_{B}^{2} X_{4}^{4}\right)=\left(U C_{T V} X_{2}\right. \\
\left.-A_{B} X_{4}^{2}\right)\left(U C T V X_{2}+A_{B} X_{4}^{2}\right)
\end{array}
$$

If $\left(U C_{T} V X_{2}-A_{B} X_{4}^{2}\right)>1$, then $U^{2} C_{T V}^{2} y_{2}>\left(U C_{T} V X_{2}+\right.$ $\left.A_{B} X_{4}^{2}\right)$, so:

$$
0<\frac{d y_{2}}{d \tau}<U^{2} C_{T V}^{2} y_{2}
$$

Assuming $y_{1}=B e^{A t}$ provides:

$$
0<B A<U^{2} C_{T V}^{2} B
$$

where $B$ must be positive, and consequently, $A$ will be positive, too. Thus, for this condition, the boundary layers of Eq. (21) are not exponentially stable. For other conditions, it can be shown that the boundary layers model is stable.

By following the same procedure, it can proved that the boundary layers of Eq. (22) is exponentially stable. So, the only concern in this analysis is associated with Eq. (21), which is not partially stable. The results of this analysis are used in section $\mathrm{V}$, where the results of the perturbed equation are discussed.

Based on the boundary layers stability analysis of the system, it is obtained that Eqs. (17) and (19) have no challenges with applying perturbation theory for control signal $U>C$. It should be noted that the boundary layers stability analysis is performed if the control signal is less than $C$, i.e., $U<-C$. By taking a similar approach as mentioned above, it can be found that the boundary layers of Eq. (17) are not unconditionally stable, thus perturbation theory cannot be applied.

In summary, it is shown that perturbation theory can only be applied to Eq. (19). This equation satisfies all necessary conditions mentioned in singular perturbation theory. In order to confirm this analysis, the theory was also applied to Eqs. (17) and (18), and it was observed that the algorithm became unstable.

\section{2) Perturbation theory applied to $P_{3}$}

Here, perturbation theory is applied to Eq. (19). So, by putting the corresponding equation off, and considering it as $\psi$ (see section III), other state variables are considered as $\phi$. According to the analysis in section III, the reduced system with the accuracy of $O(\varepsilon)$ can be used. After applying perturbation theory to the system, Eq. (19) will change. Accordingly, the perturbed equation for $U>C$ will be:

$$
P_{3}=\frac{P_{1}+\alpha_{p} P_{0}}{1+\alpha_{p}}
$$

where $\alpha_{p}=\left(\frac{U C_{P V}}{C_{C}}\right)^{2}$, and in the state space:

$$
X_{3}=\frac{X_{1}}{\sqrt{\alpha_{p}}}
$$

The above equation (Eq. 35) will be used instead of Eq. (19). The response of the perturbed equation is close to the original equation except for small time scales.

On the other hand, for $U<-C$ :

$$
P_{3}=\frac{P_{1}+\alpha_{T} P_{4}}{1+\alpha_{T}}
$$

where $\alpha_{p}=\left(\frac{U C_{T V}}{C_{C}}\right)^{2}$. Also, when $-C<U<C$, the Tikhonovs theorem results in $P_{3}=P_{1}$.

An important note that must be made here is the effect of the perturbed equation on the other circuit variables. In other words, $\bar{P}_{3}$ will be involved in other relations such as Eq. (17). In this equation, $\varepsilon \dot{P}_{1}=\left(Q_{A}+Q_{C}\right)$, in which

$$
Q_{C}=C_{C} \sqrt{P_{3}-P_{1}} \operatorname{Step}\left(P_{3}-P_{1}\right)
$$

for $U>C$. So, there is a small error between $\Delta P=P_{3}-P_{1}$, and $\Delta \bar{P}=\bar{P}_{3}-\bar{P}_{1}$. Thus, it is necessary to modify $\bar{Q}_{C}$ (volumetric flow rate in perturbed system). This modification is done by introducing a parameter multiplied by the perturbed volumetric flow rate known as a correction factor. So, considering $\bar{Q}_{C}$ to be

$$
\bar{Q}_{C}=\lambda C_{C} \sqrt{\bar{P}_{3}-\bar{P}_{1}} \operatorname{Step}\left(\bar{P}_{3}-\bar{P}_{1}\right)
$$

for $U>C$, it is rewritten as

$$
\bar{Q}_{C}=\lambda C_{C} \sqrt{\frac{\alpha_{p}}{1+\alpha_{p}}} \sqrt{P_{0}-\bar{P}_{1}} \operatorname{Step}(\Delta \bar{P}),
$$

using Eq. (35). On the other hand,

$$
d \bar{Q}_{C}=\lambda^{2} C_{C}^{2} \frac{\alpha_{p}}{1+\alpha_{p}} \operatorname{Step}^{2}(\Delta \bar{P})+\bar{Q}_{C} \frac{d \operatorname{Step}(\Delta \bar{P})}{\operatorname{Step}(\Delta \bar{P})}
$$

where $\Delta \bar{P}=\bar{P}_{3}-\bar{P}_{1}$. Next,

$$
Q_{C}=C_{C} \sqrt{P_{3}-P_{1}} \operatorname{Step}\left(P_{3}-P_{1}\right)
$$


and,

$$
d Q_{C}=\frac{C_{C}^{2}}{2 Q_{C}}\left(d P_{3}-d P_{1}\right) \operatorname{Step}^{2}(\Delta P)+Q_{C} \frac{d \operatorname{Step}(\Delta P)}{\operatorname{Step}(\Delta P)}
$$

Assuming $\bar{Q}_{C}=Q_{C}$, and $d \bar{P}_{1}=d P_{1}$, and by equating $d \bar{Q}_{C}=d Q_{C}$, allows

$$
\begin{array}{r}
\lambda^{2} C_{C}^{2} \frac{\alpha_{p}}{1+\alpha_{p}} \frac{d \bar{P}_{1}}{2 \bar{Q}_{C}} \operatorname{Step}^{2}(\Delta \bar{P})+\bar{Q}_{C} \frac{d \operatorname{Step}(\Delta \bar{P})}{\operatorname{Step}(\Delta \bar{P})}= \\
\frac{C_{C}^{2}}{2 Q_{C}}\left(d P_{3}-d P_{1}\right) \operatorname{Step}^{2}(\Delta P)+Q_{C} \frac{d \operatorname{Step}(\Delta P)}{\operatorname{Step}(\Delta P)}
\end{array}
$$

to be obtained.

Considering $\Delta P=\Delta \bar{P}+\sigma$, it can be shown that

$$
\frac{d \operatorname{Step}(\Delta P)}{\operatorname{Step}(\Delta P)}-\frac{d \operatorname{Step}(\Delta \bar{P})}{\operatorname{Step}(\Delta \bar{P})} \cong\left\{\begin{array}{rr}
0 & \Delta \bar{P} \neq 0 \\
-\sigma & \Delta \bar{P}=0
\end{array}\right.
$$

As a result,

$$
\lambda^{2} \frac{\alpha_{p}}{1+\alpha_{p}} d \bar{P}_{1} \cong\left(d P_{3}-d P_{1}\right) .
$$

Thus, to find the value of $\lambda$, a relation between $d P_{3}$, and $d P_{1}$ needs to be identified. Considering Eq. (17) and (19), we have

$$
\frac{\varepsilon_{1} d P_{1}}{\varepsilon_{2} d P_{3}}=\frac{Q_{A}+Q_{C}}{Q_{E}-Q_{C}}=\frac{1}{\gamma}
$$

when the velocity is equal to zero. Therefore, $d P_{3}=$ $\gamma \frac{\varepsilon_{1}}{\varepsilon_{3}} d P_{1}=\gamma \frac{V_{1}}{V_{3}} d P_{1}$. Finally, $\lambda^{2} \frac{\alpha_{p}}{1+\alpha_{p}} d \bar{P}_{1} \cong\left(\gamma \frac{V_{1}}{V_{3}}-1\right) d P_{1}$, and

$$
\lambda \cong \sqrt{\frac{\alpha_{p}}{1+\alpha_{p}}\left(\gamma \frac{V_{1}}{V_{3}}-1\right)}
$$

This is an estimation of the parameters affecting $\lambda$. As this relation shows, the correction factor depends on the volumes $V_{1}$, and $V_{3}$, in addition to $\alpha_{p}$. Therefore, by knowing the values of $V_{1}, V_{3}$, and $\alpha_{p}$ and estimating the constant value of $\gamma$ for one scenario, then we can use the algorithm for other scenarios.

\section{RESULTS AND DISCUSSION}

In this section, the results of the introduced hydraulic modelling approach are shown. The results also shed a light on the origin of difficulties associated with efficient computing. In addition, it is demonstrated how perturbation theory can help to overcome these problems and the conditions needed for its use.

Figure 2a shows the response of the hydraulic system under investigation in the time domain for the volume of $V_{3}=10^{-4} \mathrm{~m}^{3}$. The system is solved using the Runge-Kutta numerical integration algorithm with a fixed time step of $10^{-4}$ sec. As Fig. 2a indicates, the time response is not acceptable. The parameters and initial values of integration used in the simulation are tabulated in Table I. In Fig. $2 b$, the volume used in the calculations is increased to $V_{3}=5 \times 10^{-3} \mathrm{~m}^{3}$ where the time step is increased to $10^{-3} \mathrm{sec}$. The results demonstrate that the same problem of stability remains, as shown in Fig. 2a.

Figure 2c reveals how the results can take an anticipated trend when $V_{3}=5 \times 10^{-3} \mathrm{~m}^{3}$ and the time step is $10^{-4} \mathrm{sec}$.

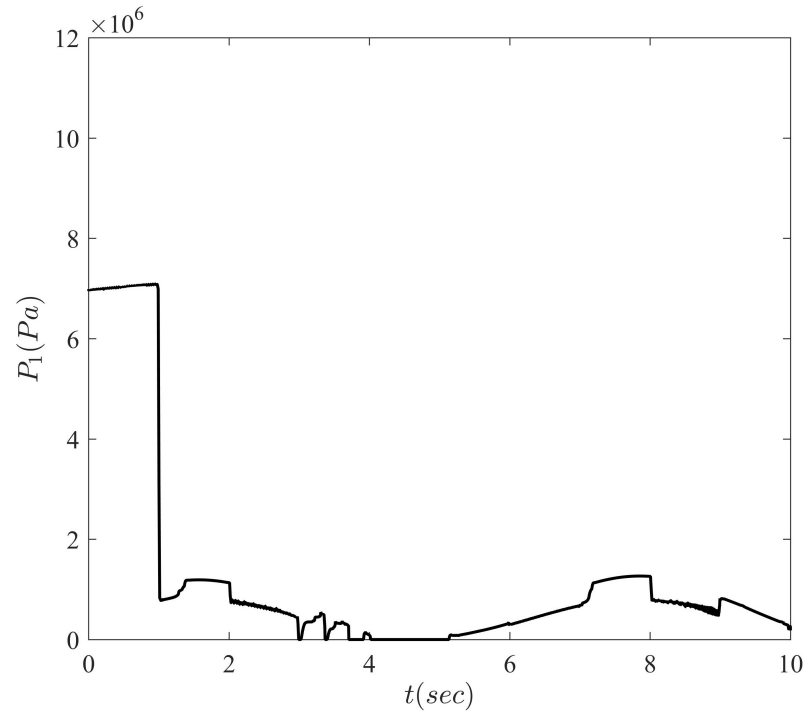

(a)

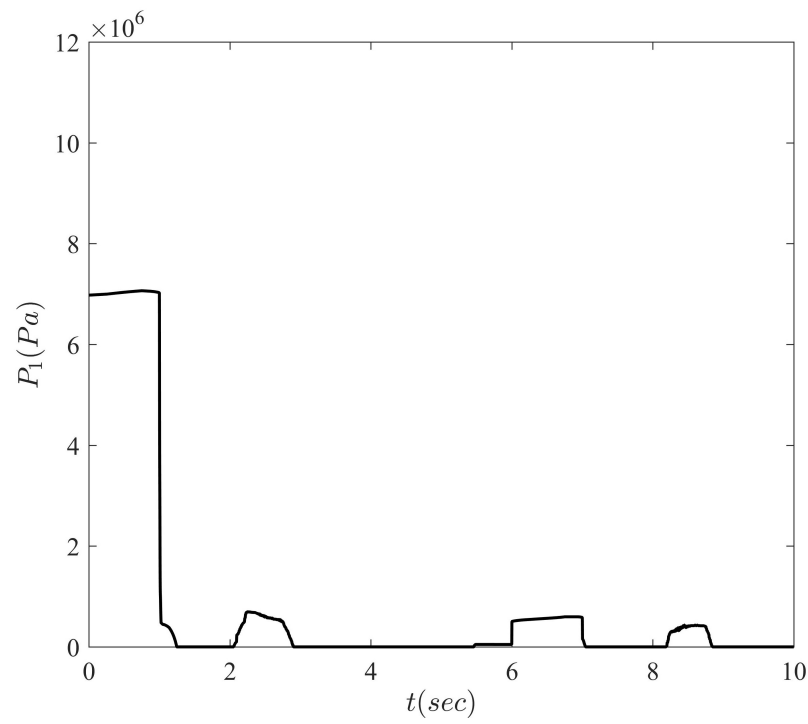

(b)

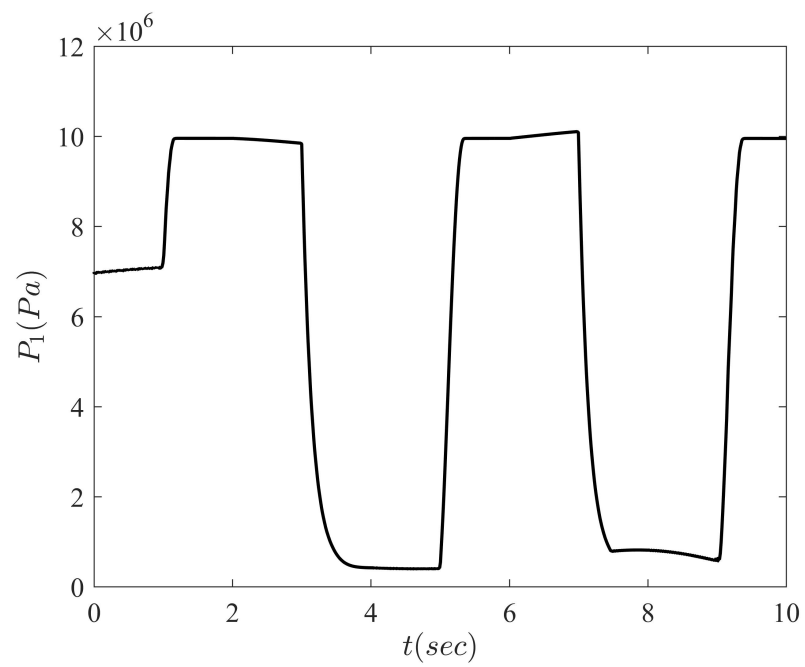

(c)

Fig. 2: Response of the original system for different conditions a) $V_{3}=10^{-4} \mathrm{~m}^{3}$, time step $=10^{-4} \mathrm{sec}$ b) $V_{3}=5 \times 10^{-3} \mathrm{~m}^{3}$, time step $=10^{-3} \mathrm{sec}$, and c) $V_{3}=5 \times 10^{-3} \mathrm{~m}^{3}$, time step $=$ $10^{-4}$ sec. 
Comparing Figs. 2a and $2 \mathrm{c}$, it can be concluded that the volume decrease of $V_{3}$ by 50 times leads to the failure in the integration of the system. On the other hand, comparing Figs. $2 b$ and $2 c$, it can be seen that all conditions are same but the time step size is greater by one order of magnitude in Fig. 2b than in Fig. 2c. These results in Fig. 2 indicate that the selection of suitable values for time step size and the volume can directly affect the stability of the solution. As small volumes occur in many applications, the choice of smaller time steps may lead to a slower computational process.

As mentioned above, a stable integration requires the time step to decrease as the volume reduces. When volume size approaches zero, the integration will eventually fail. This problem can be resolved by employing the singular perturbation theory.

Figure 3 depicts the pressures in both sides of the piston (point 1 in Fig. 3a, and point 2 in Fig. 3b) as a function of time. These values are for a perturbed system without any

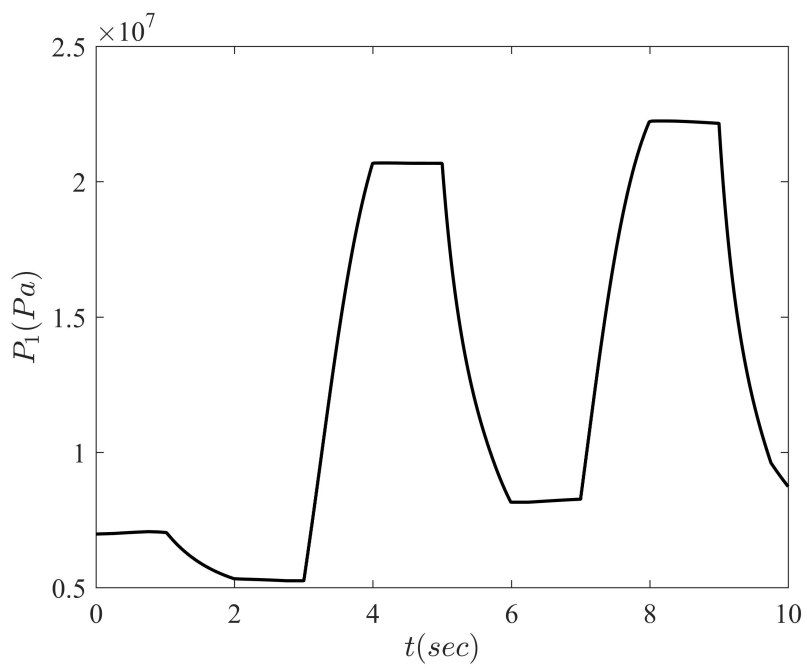

(a)

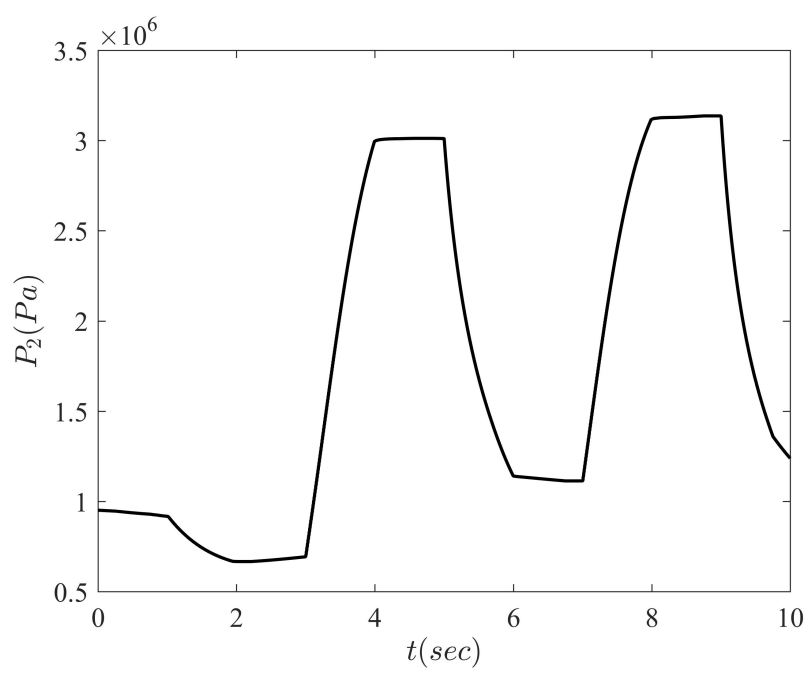

(b)

Fig. 3: Response of the perturbed system without volumetric flow rate modification with the time step of $10^{-3} \mathrm{sec}$. additional corrections and calculations. In other words, only Eqs. 35 and 37 are used instead of Eq. 19. Comparing Fig. 3a and Fig. 2c reveals that the results of the perturbed system do not match the results of the original system with a lower time step. Therefore, applying the perturbation theory alone is not enough to obtain reasonable results.

As mentioned in section IV, and demonstrated in Fig. 3, the errors associated with the volumetric flow rate $Q_{C}$ are responsible for this inconsistency. Fig. 4 presents the volumetric flow rate $Q_{C}$ in different systems, namely the original system, the perturbed system, and the perturbed system with modifications. From the figure, it can be noted that there is significant difference between the volumetric flow rate of the original system and the perturbed system, which requires modification of the perturbed system as mentioned in Sec. IV-B2.

The modification is performed by introducing a correction factor $\lambda$, which is multiplied by the volumetric flow rate. The correction factor is estimated as $\lambda \cong \frac{1+\alpha_{p}}{\alpha_{p}}\left(\gamma \frac{V_{1}}{V_{3}}-1\right)$ for $U>$ $C$ (see section IV-B2). For instance, by setting parameters as $V_{3}=2.45 \times 10^{-3} \mathrm{~m}^{3}, V_{3}=5 \times 10^{-3} \mathrm{~m}^{3}$ and by calculating $\alpha_{p}=\left(U C_{P V} / C_{c}\right)^{2}=2.56 \times 10^{-4}$ using the values given in Table A1, and assuming $\gamma=49$, the correction factor is obtained as $\lambda=300$. According to Fig. 4, results based on the modified volumetric flow rate $Q_{C}$ agree with original system. Therefore, the modified volumetric flow rate relation needs to be used to get acceptable results. It is important in terms of realistic results, however, that the volume $V_{3}$ is accounted for. This volume can be treated with the modification done on the volume flow rate, for which reason the modification done on the volumetric flow rate is essential.

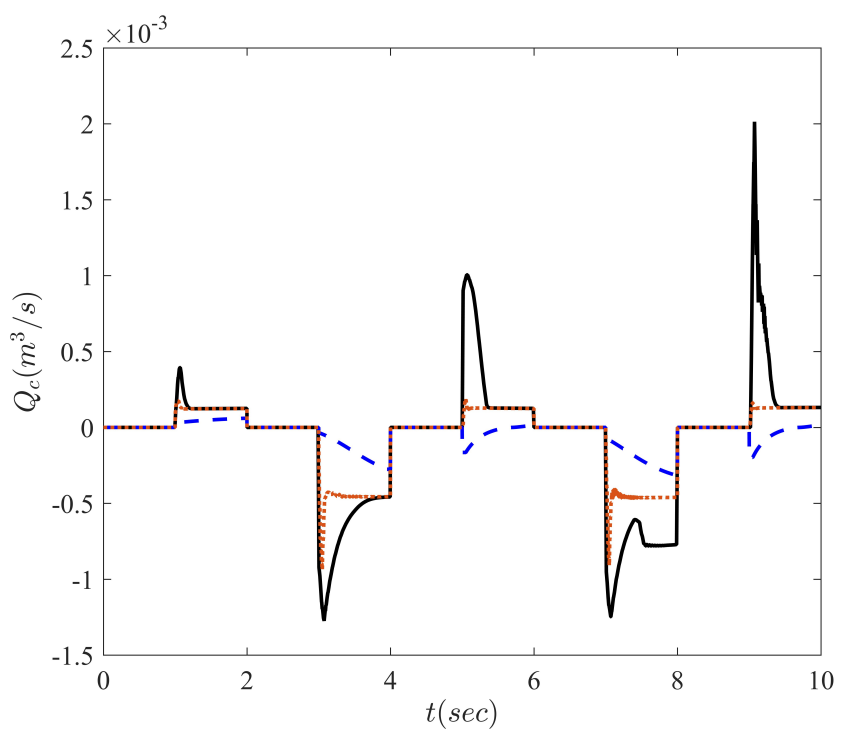

Fig. 4: Volumetric flow rate $Q_{C}$ for different systems: solid black line for the original system with $V_{3}=10^{-4} \mathrm{~m}^{3}$ and time step of $10^{-4}$ sec; dashed blue line for perturbed system without modification with time step of $10^{-3} \mathrm{sec}$; and pointed brown line for perturbed system with volumetric flow rate modification with time step of $10^{-3} \sec$ and $\gamma=49$. 


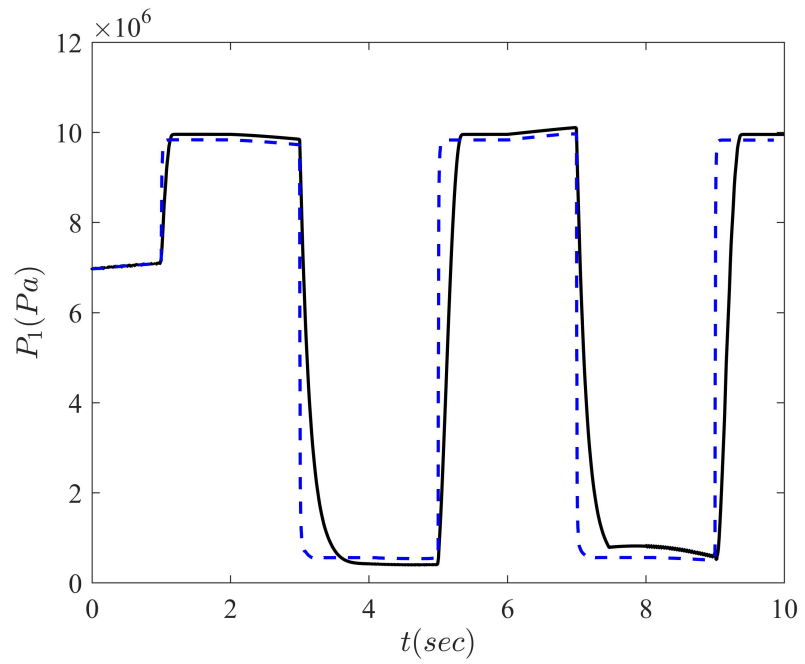

(a)

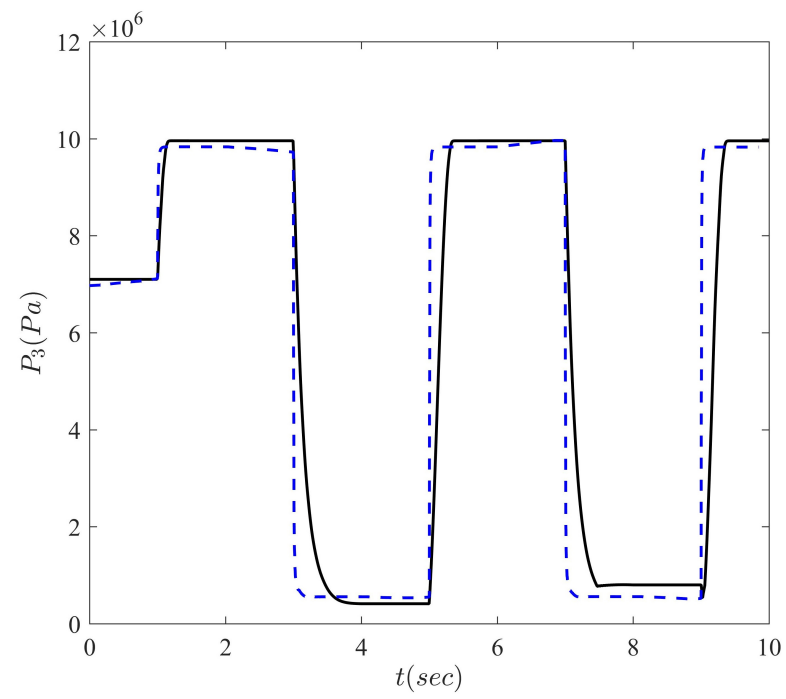

(c)

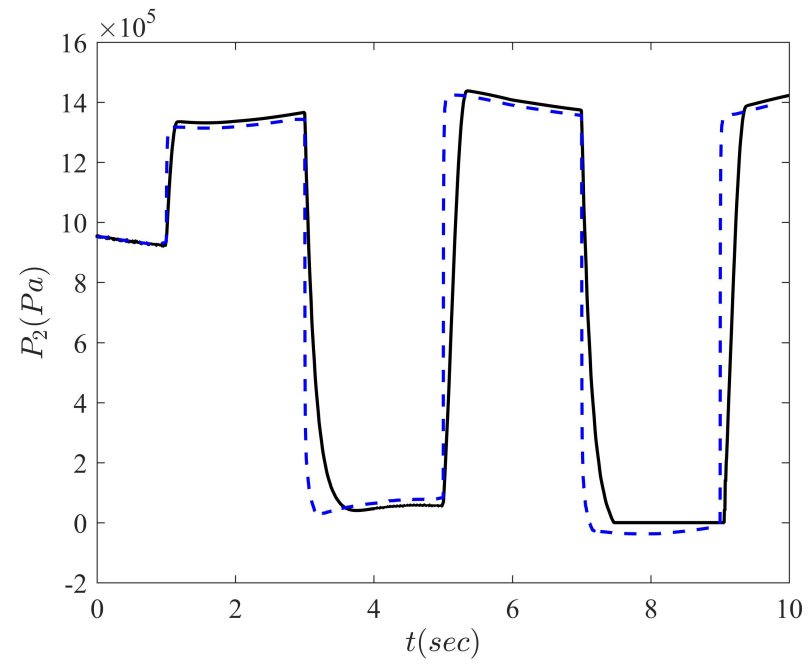

(b)

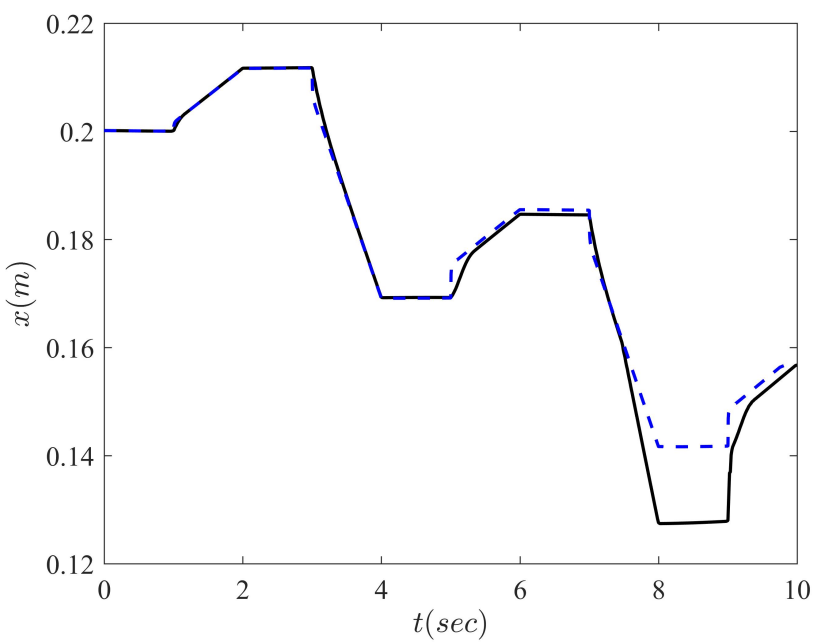

(d)

Fig. 5: Response of the original (black solid line) and perturbed system with volumetric flow rate modification (blue dash line). Variation of a) $P_{1}$, b) $P_{2}$, c) $P_{3}$, and d) $x$ with time. The original system corresponds to $V_{3}=5 \times 10^{-3} \mathrm{~m}^{3}$ and time step of $10^{-4} \mathrm{sec}$, and the perturbed system to the time step of $10^{-3} \mathrm{sec}$ and $\gamma=49$.

Figure 5 shows the results of the perturbed system with the modified volumetric flow rate (blue dash line) and the results of the original system (black solid line). Figures 5a-5d present the variations of $P_{1}, P_{2}, P_{3}$, and $x$ with time, respectively. Within the first second, the valve spool position is placed in the mid-position, where it does not let the oil flow. Both pressures and piston position remain fixed. In the next stage of time, i.e., $1<t<2 \mathrm{sec}$ the valve piston is placed at the left position, and pressures $P_{1}$ and $P_{3}$ approach the pump pressure. As a result, the piston is pushed to the right. In the next stage of time, i.e., $2<t<3 s e c$, the valve spool position is brought back to the middle position. However, the pressures on both sides of the piston with the external load are balanced by the same magnitudes as in the previous stage of $1<t<2 \mathrm{sec}$, and thus the piston position remains fixed during this stage. In the next stage, i.e., $3<t<4 \mathrm{sec}$ the valve spool goes to the right position, and consequently the pressures $P_{1}$ are $P_{3}$ are decreased near to the tank pressure. So, as Fig. 5d shows, the piston goes back to the left.

Figure 5 compares the response of the modified system based on perturbation theory against the original system in which the used time step is 10 times smaller than the one used in the modified system. As can be seen, the perturbed system agrees well with the original system. As mentioned before, the accuracy of the modeling based on perturbation mostly depends on the modification done for the volumetric flow rate. This explains the small difference in piston displacement within $8<t<9 \mathrm{sec}$. As a conclusion, the more accurate the volumetric flow rate modification, the closer is the response to the original system. It should be noted that the original system uses the time step of $10^{-4} \mathrm{sec}$ while the perturbed system is solved with the time step of $10^{-3} \mathrm{sec}$. It was observed that 
this time step could be increased up to $0.003 \mathrm{sec}$ without any stability problem.

The frequency responses of the original and perturbed system with and without volumetric flow rate correction are illustrated in Figs. 6a-6c. In this figure, the normalized Fourier transform of the volumetric flow rate in the time domain is displayed versus frequency. In Figs. $6 a$ and $6 c$, a peak occurs at the frequency of $0.25 \mathrm{~Hz}$, which is the frequency of the piston. However, Fig. $6 \mathrm{~b}$ does not display any peak at that frequency. Thus, it can be claimed that the volumetric flow rate modification reproduces same frequencies in the original and the perturbed systems.

\section{CONCLUSION}

In this paper, the singular perturbation theory is implemented as an alternative way for modeling hydraulic circuits with small volumes. First, this technique was applied to the system to show that all necessary conditions, especially the stability of boundary-layers model, were satisfied. Then, the perturbed equations were derived and implemented in other relations affected by this theory such as the volumetric flow rate in the studied case. It was shown that small volume $V_{3}$ has a significant effect on the results and must be kept in the modeling formulation. The small volume is omitted when applying perturbation theory based approach; it is however involved in the modified relation. The presented perturbation theory can speed up computation by allowing the use of larger time steps while maintaining accuracy in both the time and frequency spaces, which makes it suitable for application in real-time simulations. Future studies will be devoted to the application of this method in other hydraulic systems with different configurations as well as systems combined of several smaller systems.

\section{APPENDIX A}

The step function used in the simulation is presented in the relation (A1):

Step $=$

$$
\left\{\begin{array}{lc}
1 & \Delta P>10^{5} \\
1-2\left(3+\frac{\Delta P-10^{5}}{10^{5}}\right)\left(\frac{\Delta P-10^{5}}{2 \times 10^{5}}\right)^{2} & -10^{5}<\Delta P<10^{5} \\
-1 & -10^{5}<\Delta P
\end{array}\right.
$$

The spool position can be modeled using the following relation:

$$
\dot{U}=\frac{U_{i n}-U}{\tau}
$$

where, $U_{i n}$ is an input equal to 10 , and $\tau$ has a value of 0.1 . So, the solution of the above equation, gives $U$.

\section{ACKNOWLEDGMENT}

The authors would like to acknowledge the financial support for this work from SIM-Platform at LUT.

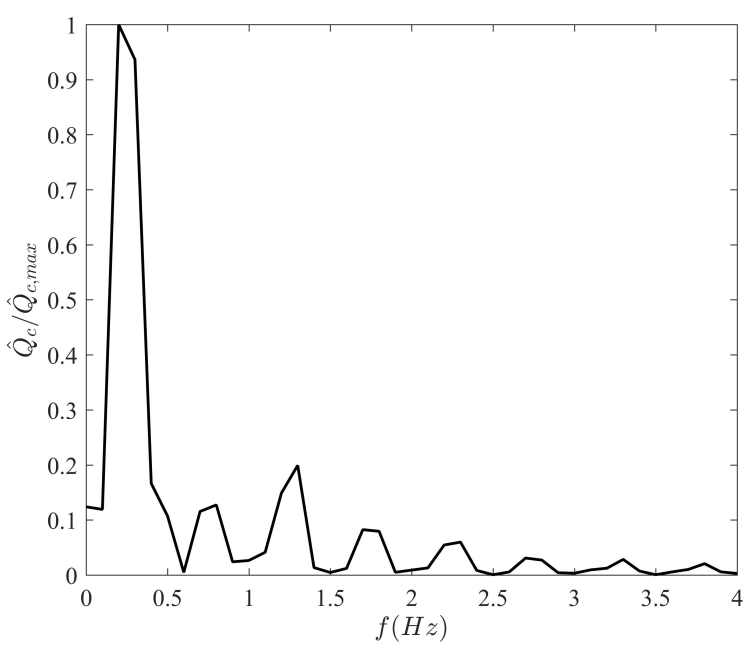

(a)

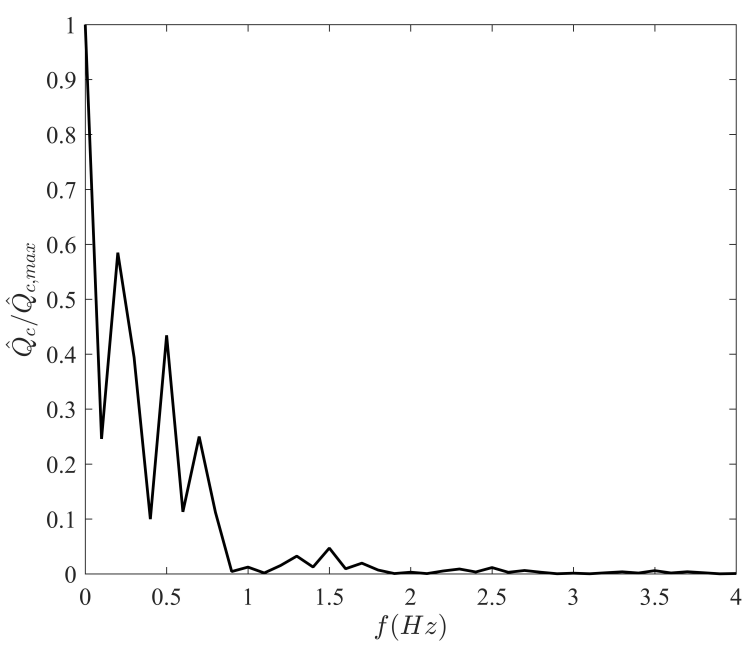

(b)

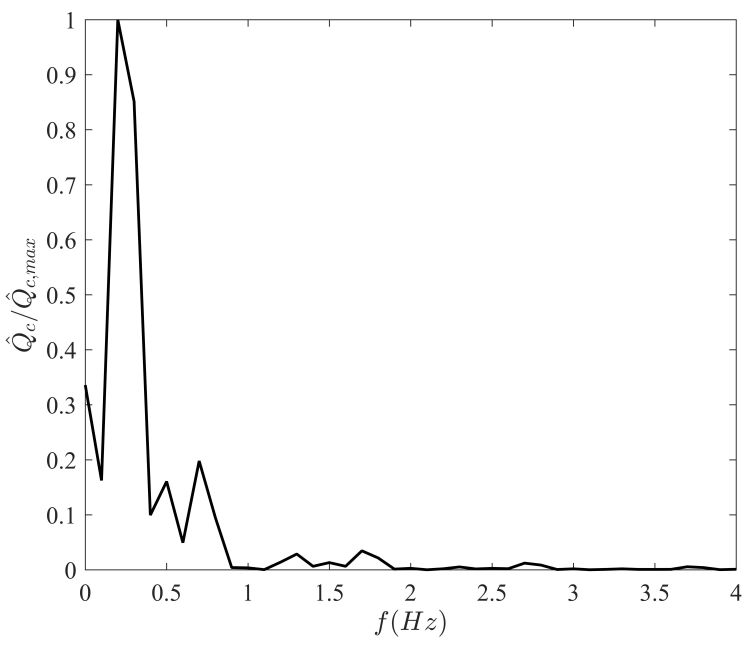

(c)

Fig. 6: Normalized Fourier transform of volumetric flow rate versus frequency: a) original system with $V_{3}=5 \times 10^{-3} \mathrm{~m}^{3}$ and time step of $10^{-4} \mathrm{sec}$; b) perturbed system without volumetric flow rate modification with time step of $10^{-3} \mathrm{sec}$; and c) perturbed system with volumetric flow rate modification with time step of $10^{-3} \mathrm{sec}$ and $\gamma=49$. 


\section{REFERENCES}

[1] C. Kaddissi, J.-P. Kenne, and M. Saad, "Identification and real-time control of an electrohydraulic servo system based on nonlinear backstepping," IEEE/ASME Transactions on Mechatronics, vol. 12, no. 1, pp. 12-22, 2007.

[2] B. Xu, R. Ding, J. Zhang, and Q. Su, "Modeling and dynamic characteristics analysis on a three-stage fast-response and large-flow directional valve," Energy conversion and management, vol. 79, pp. 187-199, 2014.

[3] F. Wang and Y. Chen, "Dynamic characteristics of pressure compensator in underwater hydraulic system," IEEE/ASME transactions on mechatronics, vol. 19, no. 2, pp. 777-787, 2014.

[4] B. Xu, R. Ding, J. Zhang, L. Sha, and M. Cheng, "Multiphysicscoupled modeling: Simulation of the hydraulic-operating mechanism for a sf6 high-voltage circuit breaker," IEEE/ASME Transactions on Mechatronics, vol. 21, no. 1, pp. 379-393, 2016.

[5] D. E. Bowns and L. M. Wang, "The digital computation of pressures in hydraulic pipes with small volume using an iterative technique," Proceedings of the Institution of Mechanical Engineers, Part C: Mechanical Engineering Science, vol. 204, no. 1, pp. 29-36, 1990.

[6] R. Piché and A. Ellman, "Numerical integration of fluid power circuit models using two-stage semi-implicit Runge-Kutta methods," Proceedings of the Institution of Mechanical Engineers, Part C: Journal of Mechanical Engineering Science, vol. 208, no. 3, pp. 167-175, 1994.

[7] W. Borutzky, B. Barnard, and J. Thoma, "An orifice flow model for laminar and turbulent conditions," Simulation Modelling Practice and Theory, vol. 10, no. 3-4, pp. 141-152, 2002.

[8] F. Pfeiffer and F. Borchsenius, "New hydraulic system modelling," Modal Analysis, vol. 10, no. 10, pp. 1493-1515, 2004.

[9] S. Esqué, A. Raneda, and A. Ellman, "Techniques for studying a mobile hydraulic crane in virtual reality," International Journal of Fluid Power, vol. 4, no. 2, pp. 25-35, 2003.

[10] W. A. Welsh, "Simulation and correlation of a helicopter air-oil strut dynamic response," in AHS, Annual Forum, 43 rd, Saint Louis, MO, Proceedings., vol. 1, 1987, pp. 231-242.

[11] F. Pfeiffer, M. Foerg, and H. Ulbrich, "Numerical aspects of non-smooth multibody dynamics," Computer Methods in Applied Mechanics and Engineering, vol. 195, no. 50, pp. 6891-6908, 2006.

[12] O. Brüls and M. Arnold, "The generalized- $\alpha$ scheme as a linear multistep integrator: toward a general mechatronic simulator," Journal of computational and nonlinear dynamics, vol. 3, no. 4, p. 41007, 2008.

[13] K. J. Bathe, "Finite Element Procedures, Prentice-Hall, Englewood Cliffs, NJ, 1996."

[14] A. Cardona and M. Geradin, "Modeling of a hydraulic actuator in flexible machine dynamics simulation," Mechanism and Machine Theory, vol. 25, no. 2, pp. 193-207, 1990.

[15] A. Ylinen, J. Mäkinen, and R. Kouhia, "Two Models for Hydraulic Cylinders in Flexible Multibody Simulations," in Computational Methods for Solids and Fluids. Springer, 2016, pp. 463-493.

[16] A. Ylinen, H. Marjamäki, and J. Mäkinen, "A hydraulic cylinder model for multibody simulations," Computers \& Structures, vol. 138, pp. 62 72, 2014.

[17] O. A. Bauchau and H. Liu, "On the modeling of hydraulic components in rotorcraft systems," Journal of the American Helicopter Society, vol. 51, no. 2, pp. 175-184, 2006.

[18] M. Naya, J. Cuadrado, D. Dopico, and U. Lugris, "An efficient unified method for the combined simulation of multibody and hydraulic dynamics: comparison with simplified and co-integration approaches," Archive of Mechanical Engineering, vol. 58, no. 2, pp. 223-243, 2011.

[19] T. R. Young and J. P. Boris, "A numerical technique for solving stiff ordinary differential equations associated with the chemical kinetics of reactive-flow problems," The Journal of Physical Chemistry, vol. 81 no. 25 , pp. 2424-2427, 1977.

[20] J. Yao, Z. Jiao, D. Ma, and L. Yan, "High-accuracy tracking control of hydraulic rotary actuators with modeling uncertainties," IEEE/ASME Transactions on Mechatronics, vol. 19, no. 2, pp. 633-641, 2014.

[21] J. Watton, Fluid power systems: modeling, simulation, analog and microcomputer control. Prentice-Hall, Inc., 1989.

[22] H. M. Handroos and M. J. Vilenius, "Flexible semi-empirical models for hydraulic flow control valves," Journal of Mechanical Design, vol. 113 , no. 3, pp. 232-238, 1991.

[23] L. Noethen and S. Walcher, "Tikhonov's theorem and quasi-steady state," Discrete and Continuous Dynamical Systems - Series B, vol. 16, no. 3, pp. 945-961, 2011.

[24] A. N. Tikhonov, "Systems of differential equations containing small parameters in the derivatives," Matematicheskii sbornik, vol. 73, no. 3, pp. 575-586, 1952.
[25] L. Wang, W. J. Book, and J. D. Huggins, "Application of singular perturbation theory to hydraulic pump controlled systems," IEEE/ASME Transactions on Mechatronics, vol. 17, no. 2, pp. 251-259, 2012.

[26] H. K. Khalil and J. W. Grizzle, Nonlinear systems. Prentice hall New Jersey, 1996, vol. 3.

[27] P. Kokotović, H. K. Khalil, and J. O'reilly, Singular perturbation methods in control: analysis and design. SIAM, 1999.

[28] L.-Y. Chen, N. Goldenfeld, and Y. Oono, "Renormalization group and singular perturbations: Multiple scales, boundary layers, and reductive perturbation theory," Physical Review E, vol. 54, no. 1, p. 376, 1996. 